\title{
50 years forward: mechanisms of hyperglycaemia-driven diabetic complications
}

\author{
Nicholas D. F. Russell ${ }^{1}$ Mark E. Cooper ${ }^{1,2}$
}

Received: 13 February 2015 / Accepted: 19 March 2015 / Published online: 24 April 2015

(C) Springer-Verlag Berlin Heidelberg 2015

\begin{abstract}
The advent of insulin treatment in 1923 meant fewer diabetes deaths from acute metabolic deterioration and sepsis and a progressive increase in the burden of disease caused by end-organ damage. These diabetic complications are the major cause of morbidity and premature mortality among diabetic subjects. Over the last 50 years it has become apparent that diabetic complications in disparate tissues may result from a combination of common pathological processes. Pathways activated by initial metabolic insults are promoted by co-factors such as renin-angiotensin-aldosterone system activation, hyperinsulinaemia, underlying genetic susceptibility, and traditional vascular risk factors, particularly hypertension and lipids. These common pathways include AGE formation, reactive oxygen species overproduction, protein kinase $\mathrm{C}$ activation, mitochondrial dysfunction and activation of proinflammatory and profibrotic signalling cascades. Once established, these interlinked pathways become self-perpetuating. Many drugs acting against individual downstream targets in these pathways have failed due to lack of efficacy or adverse effects. Gains in the future may be made by better control of existing risk factors, more sophisticated modulation of tissue glucose and insulin signalling, and interventions to improve mitochondrial function and reduce oxidative stress.
\end{abstract}

Electronic supplementary material The online version of this article (doi:10.1007/s00125-015-3600-1) contains peer-reviewed but unedited supplementary material, which is available to authorised users.

Mark E. Cooper

mark.cooper@bakeridi.edu.au

1 Department of Medicine, University of Melbourne (Austin Health), Heidelberg, VIC, Australia

2 Baker IDI Heart and Diabetes Institute, PO Box 6492, Melbourne, VIC 3004, Australia
Epigenetic and microRNA research may lead to methods to disrupt the mechanisms whereby pathological pathways are perpetuated. Expansion in capacity and expertise in biomarker measurement and analysis may allow better targeting of therapies to patients who are most likely to benefit. This is one of a series of commentaries under the banner ' 50 years forward', giving personal opinions on future perspectives in diabetes, to celebrate the 50th anniversary of Diabetologia (1965-2015).

Keywords Advanced glycation end-products (AGEs) . Cardiovascular disease - Diabetic complications .

Hyperglycaemia $\cdot$ Mitochondria $\cdot$ Nephropathy $\cdot$ Protein kinase C (PKC) · Reactive oxygen species (ROS) · Reninangiotensin-aldosterone system (RAAS) · Retinopathy

\author{
Abbreviations \\ ARB Angiotensin II receptor blocker \\ NFKB Nuclear factor $\mathrm{K}$-light chain-enhancer of activated \\ B cells \\ NOX NADPH oxidase \\ PKC Protein kinase $\mathrm{C}$ \\ $\operatorname{PPAR} \alpha \quad$ Peroxisome proliferator-activated receptor $\alpha$ \\ RAAS Renin-angiotensin-aldosterone system \\ RAGE Receptor for AGE \\ ROS Reactive oxygen species \\ VEGF Vascular endothelial growth factor
}

\section{Introduction}

Papers using the term 'diabetic complications' start to appear in the medical literature from the 1930s. By then, it was well recognised that while the advent of insulin treatment in 1923 had prevented many deaths from acute metabolic deterioration 
and sepsis, the risk of 'degenerative and middle-life complications', particularly vascular disease, had become more prominent [1]. With ongoing improvements in diabetes detection and therapies to treat hyperglycaemia, the acute metabolic emergencies have continued to recede in importance. Nevertheless, patients with all forms of diabetes remain at risk of secondary injury to the vasculature, nervous system, kidney and retina. These sites of endorgan dysfunction are collectively referred to as diabetic complications.

Diabetic complications are the major cause of morbidity and premature mortality among diabetic patients. Improvements in management over the last 50 years have reduced diabetic complication rates, with the consequence that the life expectancy for individuals with either type 1 diabetes or type 2 diabetes has increased. However, the increasing prevalence of diabetes is driving an increasing total burden of morbidity and premature mortality from diabetic complications [2]. Cardiovascular disease represents the major cause of diabetes-related death, yet it is the presence or absence of nephropathy and albuminuria that most powerfully predicts mortality in modern cohorts of both type 1 and type 2 diabetes. Indeed, the aetiology of cardiovascular disease in diabetes is likely to be multifactorial, with some of the risk unrelated to conventional risk factors. In particular, insulin resistance and hyperinsulinaemia are considered potential risk factors for cardiovascular disease independent of glycaemia. Furthermore, insulin treatment per se has been postulated to enhance the risk of cardiovascular disease, although this was not observed in the Outcome Reduction with an Initial Glargine Intervention (ORIGIN) trial [3].

Landmark clinical trials, including the DCCT in type 1 diabetes and the UK Prospective Diabetes Study (UKPDS) in type 2 diabetes, and their follow-up studies confirmed the results of early observational studies showing that all major diabetic complications are to some extent dependent on diabetes duration and degree of glycaemic control [4-6]. Improvements in the management of hyperglycaemia have been extremely important in reducing the burden of diabetic complications, although tight glycaemic control has been relatively disappointing in terms of prevention of cardiovascular disease [7]. In addition, antihypertensive therapy, including inhibitors of the renin-angiotensin-aldosterone system (RAAS), lipid-lowering agents and antithrombotics, as well as multifactorial intervention approaches involving a combination of all these therapeutic modalities [8], have been shown to reduce diabetic complication rates. Despite substantial progress, currently available therapies remain insufficient to prevent diabetic complications, and it is therefore crucial to continue to investigate the mechanisms responsible for these complications.

\section{Scope of diabetic complications}

Diabetic complications have historically been divided into macrovascular and microvascular complications. Macrovascular complications result from arterial endothelial and smooth muscle inflammation and dysfunction leading to accelerated atherosclerosis, with resultant ischaemic heart disease, cerebrovascular disease and peripheral vascular disease. Microvascular complications affect small vessels of the retina, kidney and nerves, with complications resulting from impaired autoregulation of blood flow, altered permeability, inflammation, extracellular matrix accumulation, hypoxia, cell loss, neovascularisation and fibrosis.

The recognised scope of diabetic complications has continued to expand. Diabetes accelerates the progress of many agerelated and degenerative conditions. Diabetic autonomic neuropathy is associated with gastrointestinal motility disorders, erectile and bladder dysfunction, diaphoresis and orthostatic intolerance, as has been extensively described in the literature since the 1960s. Diabetes also promotes joint immobility, obstructive sleep apnoea, certain skin conditions, cardiomyopathy and cognitive decline. Recently, the term 'diabetic encephalopathy' has been proposed, encompassing cognitive dysfunction ranging from subtle reproducible deficits on neurocognitive testing in young patients with type 1 diabetes to brain atrophy and increased risk of Alzheimer's disease and vascular dementia in older diabetes patients [9].

\section{Mechanisms of complications: the current paradigm}

Current knowledge of the mechanisms of diabetic complications has recently been extensively reviewed [10]. Over the last 50 years it has become apparent that diabetic complications in disparate tissues may result from combinations of common pathological processes. The defining metabolic perturbation of diabetes is hyperglycaemia, accompanied by glycaemic variability although the relevance of glycaemic variability per se in the pathogenesis of diabetic complications remains controversial [11]. Pathways activated by these metabolic insults are amplified in tissues prone to diabetic complications by insults from aetiological co-factors, including ageing, dyslipidaemia, smoking, hyperinsulinaemia and hypertension. The end result is the tissue-specific activation of proinflammatory and profibrotic cytokines, resulting in the characteristic pathological and clinical abnormalities (Fig. 1).

Although the scope of diabetic complications seems vast, not all cell populations are prone to complications. Those affected include vascular endothelia, renal mesangial and proximal tubular cells, glomerular epithelial cells (podocytes), neurons and glial cells. In these tissues, facilitated diffusion of glucose occurs in an insulin-independent manner via the glucose transporter GLUT1 [12]. The resultant intracellular 


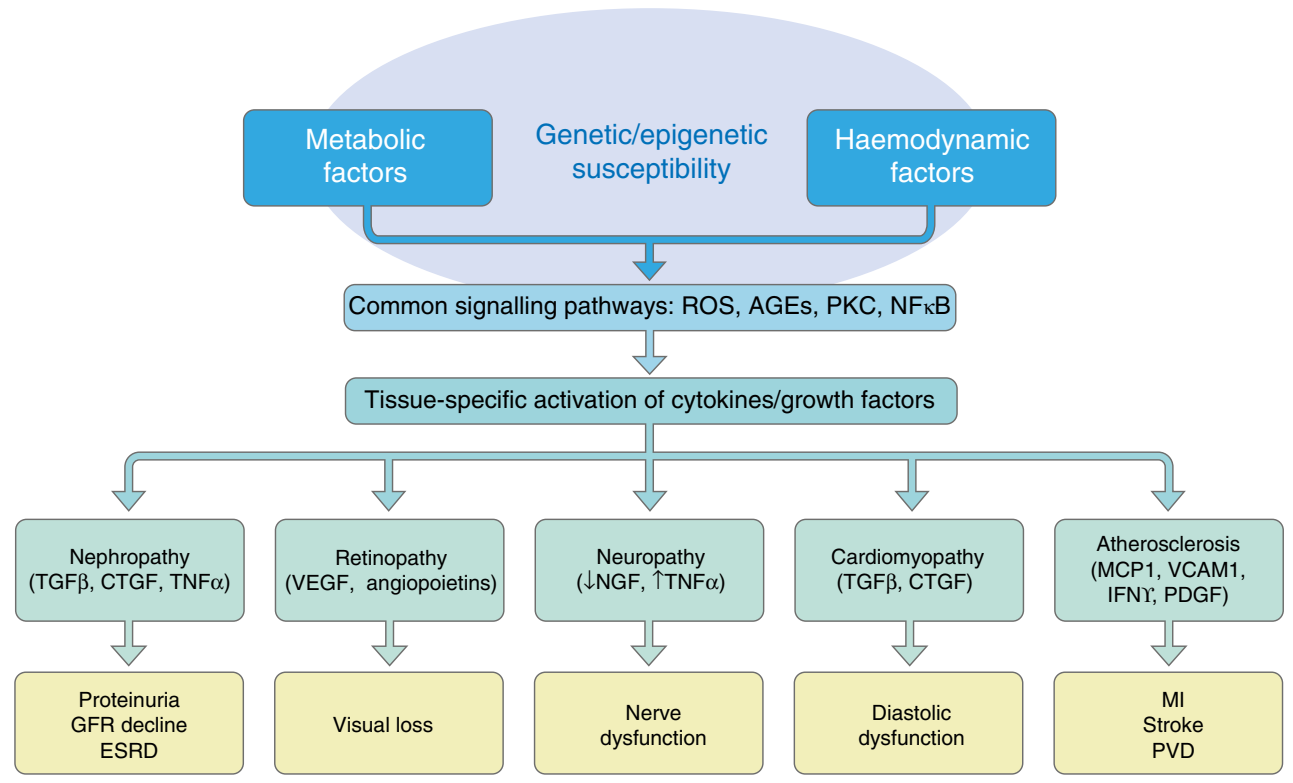

Fig. 1 Mechanisms of diabetic complications. Diabetic complications may result from a combination of common pathological processes. Pathways activated by initial metabolic insults, are promoted by co-factors such as hypertension and RAAS activation, underlying genetic susceptibility, and traditional and non-traditional vascular risk factors. These pathways result in tissue-specific activation of proinflammatory and

hyperglycaemia seems to be a key initiating factor in the development of diabetic complications.

As originally hypothesised by Brownlee [13], intracellular hyperglycaemia in diabetic complication-prone cells results in excess respiration from glucose metabolites, directly leading to increased mitochondrial superoxide production. The increase in reactive oxygen species (ROS) can overwhelm antioxidant defences, damage structures including cellular and mitochondrial DNA, and has been causally linked to activation of protein kinase $\mathrm{C}$ (PKC) isoforms and increased flux into the hexosamine and polyol pathways. These processes activate a number of proinflammatory and profibrotic intracellular signalling cascades via key redox-sensitive transcription factors such as nuclear factor $\kappa \mathrm{B}(\mathrm{NF} \kappa \mathrm{B})$. The Brownlee hypothesis is an area of ongoing controversy and active investigation. A recent study has suggested that this excess of mitochondrial ROS may not be a universal finding [14], and there is increasing evidence of the importance of nonmitochondrial sources of ROS, including cytosolic production of ROS by NADPH oxidase [15]. Furthermore, although the polyol pathway has now been under active investigation for over 40 years, except for some limited data in neuropathy, agents that inhibit this pathway, such as aldose reductase inhibitors, have not been widely used in clinical practice. Similarly, the PKC- $\beta$ inhibitor ruboxistaurin has been extensively investigated, but unimpressive clinical results have led to the decision not to progress this drug at this stage (Table 1).

There is increasing evidence that mitochondrial dysfunction may play a key role in the development of diabetic profibrotic cytokines, causing the characteristic pathological and clinical abnormalities. CTGF, connective tissue growth factor; ESRD, end-stage renal disease; GFR, glomerular filtration rate; MCP1, monocyte chemoattractant protein 1; MI, myocardial infarction; NGF, nerve growth factor; PDGF, platelet derived growth factor; PVD, peripheral vascular disease; VCAM1, vascular adhesion molecule 1

complications [10]. Excess respiration from glucose, through production of ROS, is thought to lead to mitochondrial DNA damage, electron transport chain enzyme dysfunction and upregulation of uncoupling proteins. This leads to inefficient oxidative phosphorylation, resulting in loss of mitochondrial membrane potential and reduced ATP production, as well as an increased tendency towards ROS production. Failure of mitochondrial energy production has been demonstrated in cardiomyopathy, nephropathy and neuropathy [16]. This is thought to lead to increased selective mitochondrial autophagy, resulting in decreased mitochondrial density, failure of energy-dependent cellular functions and activation of cell death pathways.

Intracellular hyperglycaemia also results in intracellular production of AGEs, a process that may also be driven by excess ROS formation. AGE-modification of proteins is pathogenic because it can disrupt intracellular enzymes and transcription factors. AGEs diffuse extracellularly and disrupt the function of extracellular matrix proteins. AGEs also act via a range of receptors, including receptor for AGE (RAGE) to further increase NFKB in diabetic complication-prone cells. This area of research, although under active investigation for over 30 years, has not yet led to any drugs that have entered clinical practice. A range of drugs, including the AGE inhibitor aminoguanidine (Table 1), have failed in clinical trials as a result of side effects or limited efficacy. Nevertheless, there remain ongoing trials with other AGE inhibitors, such as pyridoxamine (Table 1). Furthermore, alternative strategies to modulate the AGE pathway are currently under active 
Table 1 Examples of novel agents to target pathways of diabetic complications

\begin{tabular}{|c|c|c|c|c|}
\hline \multirow[t]{2}{*}{ Modifiable pathway or target } & \multirow[t]{2}{*}{ Complication } & \multicolumn{3}{|l|}{ Example agent } \\
\hline & & Under clinical investigation & In current clinical use & $\begin{array}{l}\text { Abandoned as a result of } \\
\text { lack of efficacy or } \\
\text { adverse effects }\end{array}$ \\
\hline $\begin{array}{l}\text { AGE/RAGE pathway } \\
\text { (including intermediates } \\
\text { such as methylglyoxal) }\end{array}$ & All & Pyridoxamine & & $\begin{array}{l}\text { Soluble RAGE } \\
\text { Alagebrium } \\
\text { Aminoguanidine }\end{array}$ \\
\hline Protein kinase $\mathrm{C}$ & $\begin{array}{l}\text { Retinopathy } \\
\text { Nephropathy } \\
\text { Neuropathy }\end{array}$ & & & Ruboxistaurin \\
\hline Vitamin D receptor & Nephropathy & Paricalcitol & & \\
\hline Nrf2 & Nephropathy & & & Bardoxolone methyl \\
\hline NADPH oxidase & $\begin{array}{l}\text { Retinopathy } \\
\text { Nephropathy } \\
\text { Atherosclerosis }\end{array}$ & GKT137831 (NOX1/4 inhibitor) & & \\
\hline Kallikrein-kinin system & Retinopathy & KVD001 (plasma kallikrein inhibitor) & & \\
\hline VEGF & Retinopathy & VEGF antibodies & VEGF antibodies & \\
\hline $\operatorname{PPAR} \alpha$ & Retinopathy & & Fenofibrate & \\
\hline Polyol pathway & Neuropathy & & & Aldose reductase inhibitors \\
\hline Endothelin system & $\begin{array}{l}\text { Nephropathy } \\
\text { ?Atherosclerosis }\end{array}$ & Atrasentan & & Avosentan \\
\hline Renin inhibition & Nephropathy & & & Aliskiren \\
\hline Mineralocorticoid antagonists & $\begin{array}{l}\text { Nephropathy } \\
\text { Heart failure }\end{array}$ & Finerenone & $\begin{array}{l}\text { Spironolactone } \\
\text { Eplerenone }\end{array}$ & \\
\hline Neutral endopeptidase & Heart failure & $\begin{array}{l}\text { LCZ696 (valsartan and } \\
\text { sacubitril in combination) }\end{array}$ & & Omapatrilat \\
\hline SGLT2 & $\begin{array}{l}\text { Nephropathy } \\
\text { Cardiovascular disease }\end{array}$ & SGLT2 inhibitors $^{\mathrm{a}}$ & SGLT2 inhibitors $^{\mathrm{a}}$ & \\
\hline Xanthine oxidase & Nephropathy & $\begin{array}{l}\text { Allopurinol } \\
\text { Febuxostat }\end{array}$ & & \\
\hline Phosphodiesterase & Nephropathy & Pentoxifylline & & \\
\hline $\mathrm{TGF} \beta / \mathrm{TNF} \alpha$ & Nephropathy & LY2382770 (TGF $\beta$ antibody) & & Pirfenidone \\
\hline JAK-STAT pathway & Nephropathy & Baricitinib & & \\
\hline Mitochondrial dysfunction & $\begin{array}{l}\text { Retinopathy } \\
\text { Neuropathy } \\
\text { Heart Failure }\end{array}$ & $\begin{array}{l}\text { MitoQ } \\
\text { Bendavia }\end{array}$ & & \\
\hline MCP1 & $\begin{array}{l}\text { Atherosclerosis } \\
\text { Nephropathy }\end{array}$ & $\begin{array}{l}\text { CCX140-B (MCP1 } \\
\text { receptor/CCR2 antagonist) }\end{array}$ & & \\
\hline CTGF & $\begin{array}{l}\text { Cardiomyopathy } \\
\text { Nephropathy }\end{array}$ & & & FG-3019 (CTGF antibody) \\
\hline
\end{tabular}

Many novel agents targeting individual pathways leading to diabetic complications have failed in clinical trials

For references, see ESM Table 1

${ }^{a}$ The SGLT2 inhibitors empagliflozin, canagliflozin and dapagliflozin are in clinical use as glucose-lowering agents. Further investigation is underway to determine a possible role in prevention of diabetic nephropathy and cardiovascular disease

CCR2; C-C chemokine receptor 2; CTGF, connective tissue growth factor; JAK, Janus kinase; MCP1, monocyte chemoattractant protein 1; NEP, neutral endopeptidase; Nrf2, nuclear factor (erythroid-derived 2)-like 2; SGLT2, sodium-glucose co-transporter 2; STAT, signal transducers and activators of transcription

investigation, including approaches to target AGE intermediates such as methylglyoxal and strategies to target AGE receptors such as RAGE (Table 1).

Activation of the RAAS is of particular importance in the development of diabetic complications. Although it is not clear whether the RAAS is overactivated systemically in diabetic patients prior to the development of complications, angiotensin II and aldosterone combine with hyperglycaemia to amplify downstream inflammatory and fibrotic pathways. The importance of RAAS antagonism in the prevention of nephropathy was established by the early 1990s in type 1 diabetes and by the early 2000s in type 2 diabetes. Attempts at more complete blockade of the RAAS have been unsuccessful because of side effects associated with dual ACE 
inhibitor and ARB therapy and with the addition of renin inhibitors or mineralocorticoid receptor antagonists to ACE inhibitors or ARBs (for example, see [17]). Recent research has uncovered new dimensions of the RAAS, including pathways that produce vasoactive angiotensins other than angiotensin II and perhaps an important role for the type 2 angiotensin receptor subtype in the transduction of signals leading to diabetic complications [10]. Interlinked with the RAAS is the endothelin system. Endothelin signalling in the kidney has been shown to be upregulated by hyperglycaemia and angiotensin II, and augments inflammatory and fibrotic pathways. Indeed, endothelin antagonists may afford end-organ protection in diabetes, but their clinical development has been limited since these agents cause fluid retention. Nevertheless, newer endothelin antagonists with a better side effect profile are currently in Phase 3 clinical trials for diabetic nephropathy (Table 1).

In the field of diabetic retinopathy, major progress was made over 30 years ago with benefits demonstrated with laser photocoagulation, initially for proliferative retinopathy and subsequently for macular oedema. More recently, various approaches to inhibit the biological actions of the proangiogenic growth factor known as vascular endothelial growth factor (VEGF) have been shown to reduce vision-threatening retinopathy (Table 1). Newer approaches to inhibit VEGF action as well as targeting other angiogenic cytokines and the kallikrein-kinin system are currently being investigated at the preclinical level as well as in Phase 1 and 2 clinical trials (Table 1). Interestingly, in both the Fenofibrate Intervention and Event Lowering in Diabetes (FIELD) and Action to Control Cardiovascular Risk in Diabetes (ACCORD) lipid studies, the peroxisome proliferator-activated receptor $\alpha$ $(\operatorname{PPAR} \alpha)$ agonist fenofibrate was associated with a reduction in retinopathy (Table 1). Whether this retinoprotective effect relates to an action on PPAR $\alpha$ or an anti-inflammatory action via transactivation of other receptors has not been clarified and warrants further investigation.

\section{What might the future hold?}

Multiple interlinked self-perpetuating pathways Over recent decades it has become evident that the pathways leading to diabetic complications are complex, interlinked and selfperpetuating after the initial metabolic insult. Once established, there appears to be no one single common, pharmacologically modifiable pathway, which is necessary for the development of diabetic complications. Multiple drugs targeting downstream pathways that lead to diabetic complications have been tested. Despite some modest protective effects, none have reached mainstream clinical use. Many of the targetable pathways of inflammation and fibrosis are intrinsic to host energy production and immune defence, making side effects of effective agents highly likely. The extensive interlinking of diabetic complication mechanisms introduces redundancy, which means that targeting one pathway is unlikely to be effective. On the other hand, given that key upstream events are similar, future clinical benefits of any new agent in one organ may be able to be extrapolated to other tissues that are prone to diabetic complications.

Better control of glucose and other risk factors with new and existing drugs Early tight glycaemic control and multifactorial intervention are already proven to prevent or retard diabetic complications [8]. Therefore, while developing agents to target new pathways, further gains are likely to be achieved by optimising known risk factors for diabetic complications, such as better glucose-lowering drugs, which may prove to have additional renoprotective properties without promoting hypoglycaemia or weight gain. Additional blood pressure-lowering drugs, including newer mineralocorticoid antagonists such as finerenone with potentially less risk of hyperkalaemia, are also on the horizon (Table 1). More effective lipid-lowering agents, including proprotein convertase subtilisin kexin 9 (PCSK9) inhibitors are also at an advanced stage of clinical development. However, the roles and costs of these drugs need to be considered, particularly since statins effectively reduce cholesterol in the vast majority of diabetic individuals and confer significant cardiovascular protection in this population.

Prevention of glucose uptake into cells Future progress may be made with combinations of agents that attack multiple downstream pathways or with agents that prevent the early initiation of damage by reducing intracellular hyperglycaemia. Further elucidation of glucose transporters and their functions could lead to strategies to selectively modify glucose uptake to prevent intracellular hyperglycaemia in diabetic complicationprone tissues. Drugs that inhibit sodium-glucose cotransporter 2 (SGLT2) in the renal proximal tubule are already in clinical use (Table 1). These are designed to lower plasma glucose by promoting glycosuria, but there are encouraging signs that they may also have renoprotective effects, perhaps by lowering the elevated glucose concentrations in the proximal tubular cell, as well as having other relevant actions, including blood pressure reduction and weight loss. Tissue selectivity would be a prerequisite for any attempt to modulate tissue glucose uptake in humans because reduced blood-brain barrier glucose flux via GLUT1 is implicated in the development of neurodegenerative conditions such as Alzheimer's disease [18].

More sophisticated modulation of mitochondrial function and ROS With increasing evidence that ROS play a role in diabetic complications, more selective approaches to target the various sources of ROS may be worth exploring. This focus 
on selectivity is critical because of the clinical failure of previous trials using relatively non-selective 'antioxidant' compounds, such as certain vitamins to prevent diabetic complications. More sophisticated means of modulating ROS production may be useful, for example, via inhibition of NADPH oxidase (NOX). Indeed, recent animal studies using NOX1 and NOX4 knockout mice or specific pharmacological inhibitors have demonstrated that these NOX isoforms are key transducers of diabetic vascular disease and nephropathy via ROS-mediated pathways [15]. Whether these findings translate to humans remains to be determined, but a trial is underway using the specific NOX1/NOX4 inhibitor GKT137831 in type 2 diabetic individuals with albuminuria (Table 1). Pathological roles for NOX2 and NOX5 have also been proposed, although NOX2 is unlikely to be a therapeutic target given its importance in neutrophil function.

The realisation that failure of mitochondrial energy production and reduction in mitochondrial density via reduced biogenesis or increased autophagy occurs in many tissues affected by diabetic complications, has led to the investigation of many agents that may support mitochondrial function [16]. However, drugs that specifically target mitochondria have been difficult to develop, although promising preclinical data using such agents as MitoQ and Bendavia have recently been published (Table 1).

Pathways of perpetuation Studies have clearly shown that pathways resulting in diabetic complications are selfperpetuating once activated by initial metabolic insults. It is important to unravel the mechanisms underlying this selfperpetuation if we are to successfully intervene and prevent diabetic complications. Epigenetic modifications [19] and alterations in microRNA expression patterns [20] resulting in changes in transcription and post-translational modification of proteins have been highlighted as key mechanisms whereby diabetic complication pathways are perpetuated. Furthermore, it is likely that elucidation of the epigenome will explain some of the ethnic and familial clustering of complications that has eluded genome-wide association studies. New therapeutic options with agents that erase epigenetic modifications and alter microRNA expression hold promise, but as yet there is no convincing evidence that these findings can be translated into specific treatments in humans.

\section{Diabetes pathophysiology pathways may be relevant to} complications Over the coming years, there is likely to be growing evidence that pathways relevant to the pathophysiology of diabetes are also relevant to its associated complications. The concept of pancreatic beta cell glucotoxicity is well recognised, and diabetes is characterised by a deficiency of insulin signalling which itself may contribute to diabetic complications [10]. Insulin signalling within the podocyte has been demonstrated in insulin receptor knockout studies to be important in maintaining the glomerular filtration barrier [21], and albuminuria can occur in insulin-resistant euglycaemic patients. Insulin signalling also has neurotrophic actions, prevents NEFA flux from adipocyte to arterial endothelium, and reduces autophagosome-dependent degradation of cellular organelles including mitochondria [22]. While still controversial, it has been postulated there may also be organ-selective insulin resistance in diabetes, with the resultant hyperinsulinaemia and persistent insulin signalling being deleterious in those organs that remain sensitive to insulin [10].

Technological advances and biomarkers Another emerging theme in diabetic complications is the advent of new biomarkers to better predict the development of complications and benefit from therapies. Not every patient with diabetes will experience every complication. Although albuminuria and declining estimated glomerular filtration rate are excellent predictors of subsequent end-stage renal disease, newer biomarkers that add to risk prediction for this and other complications would be helpful. Biomarkers such as tissue AGE accumulation, serum or urinary miRNA profiles, soluble $\mathrm{TNF} \alpha$ receptors, or even vascular gene expression profiles, may ultimately be useful in predicting diabetic complications in individual patients. With the marked expansion in capacity and expertise in proteomics, metabolomics, lipidomics, genomics and epigenomics, it is hoped that we will better identify those patients at risk of diabetic complications and then use pharmacogenomic approaches to target therapies to those patients who are most likely to benefit from these treatments. However, this hope might be misplaced as there is as yet no evidence that this form of precision medicine is worth pursuing or will be cost-effective in diabetes.

Duality of interest NDFR has no conflicts of interest. MEC has received honoraria and is a member of advisory boards for companies who manufacture some of the agents that are included in this review. These include AbbVie, AstraZeneca, Boehringer-Ingelheim, Eli-Lilly, MSD, Novo-Nordisk and Servier.

Contribution statement Both authors were responsible for drafting the article and revising it critically for important intellectual content. Both authors approved the version to be published.

\section{References}

1. Fletcher AA, Graham JW (1939) Complications of diabetes mellitus with special reference to cause and prevention. CMAJ 41:566-570

2. Gregg EW, Li Y, Wang J et al (2014) Changes in diabetes-related complications in the United States, 1990-2010. N Engl J Med 370: $1514-1523$

3. Gerstein HC, Bosch J, Dagenais GR et al (2012) Basal insulin and cardiovascular and other outcomes in dysglycemia. N Engl J Med 367:319-328 
4. Holman RR, Paul SK, Bethel MA et al (2008) 10-year follow-up of intensive glucose control in type 2 diabetes. N Engl J Med 359: $1577-1589$

5. de Boer IH, Sun W, Gao X et al (2014) Effect of intensive diabetes treatment on albuminuria in type 1 diabetes: long-term follow-up of the diabetes control and complications trial and epidemiology of diabetes interventions and complications study. Lancet Diabetes Endocrinol 2:793-800

6. Nathan DM, Cleary PA, Backlund JY et al (2005) Intensive diabetes treatment and cardiovascular disease in patients with type 1 diabetes. N Engl J Med 353:2643-2653

7. Zoungas S, Chalmers J, Neal B et al (2014) Follow-up of bloodpressure lowering and glucose control in type 2 diabetes. $\mathrm{N}$ Engl $\mathrm{J}$ Med 371:1392-1406

8. Gaede P, Lund-Andersen H, Parving H-H, Pedersen O (2008) Effect of a multifactorial intervention on mortality in type 2 diabetes. N Engl J Med 358:580-591

9. Sima AAF (2010) Encephalopathies: the emerging diabetic complications. Acta Diabetol 47:279-293

10. Forbes JM, Cooper ME (2013) Mechanisms of diabetic complications. Physiol Rev 93:137-188

11. Ceriello A, Kilpatrick ES (2013) Glycemic variability: both sides of the story. Diabetes Care 36:S272-S275

12. Heilig CW, Concepcion LA, Riser BL, Freytag SO, Zhu M, Cortes $P$ (1995) Overexpression of glucose transporters in rat mesangial cells cultured in a normal glucose milieu mimics the diabetic phenotype. J Clin Invest 96:1802-1814
13. Brownlee M (2001) Biochemistry and molecular cell biology of diabetic complications. Nature 414:813-820

14. Dugan LL, You Y-H, Ali SS et al (2013) AMPK dysregulation promotes diabetes-related reduction of superoxide and mitochondrial function. J Clin Invest 123:4888-4899

15. Gray SP, Di Marco E, Okabe J et al (2013) NADPH oxidase 1 plays a key role in diabetes mellitus-accelerated atherosclerosis. Circulation 127:1888-1902

16. Bennett GJ, Doyle T, Salvemini D (2014) Mitotoxicity in distal symmetrical sensory peripheral neuropathies. Nat Rev Neurol 10: 326-336

17. Fried LF, Emanuele N, Zhang JH (2013) Combined angiotensin inhibition for the treatment of diabetic nephropathy. N Engl J Med 369:1892-1903

18. Winkler EA, Nishida Y, Sagare AP et al (2015) GLUT1 reductions exacerbate Alzheimer's disease vasculo-neuronal dysfunction and degeneration. Nat Neurosci. doi:10.1038/nn.3966

19. Cooper ME, El-Osta A (2010) Epigenetics: mechanisms and implications for diabetic complications. Circ Res 107:1403-1413

20. Kato M, Castro NE, Natarajan R (2013) MicroRNAs: potential mediators and biomarkers of diabetic complications. Free Radic Biol Med 64:85-94

21. Welsh GI, Hale LJ, Eremina V et al (2010) Insulin signaling to the glomerular podocyte is critical for normal kidney function. Cell Metab 12:329-340

22. Higgins GC, Coughlan MT (2014) Mitochondrial dysfunction and mitophagy: the beginning and end to diabetic nephropathy? $\mathrm{Br} \mathrm{J}$ Pharmacol 171:1917-1942 